\title{
Approximate Solutions to Time-Fractional Schrödinger Equation via Homotopy Analysis Method
}

\author{
Najeeb Alam Khan, ${ }^{1}$ Muhammad Jamil, ${ }^{2,3}$ and Asmat Ara ${ }^{1}$ \\ ${ }^{1}$ Department of Mathematics, University of Karachi, Karachi 75270, Pakistan \\ ${ }^{2}$ Abdul Salam School of Mathematical Sciences, GC University, Lahore, Pakistan \\ ${ }^{3}$ Department of Mathematics, NED University of Engineering and Technology, Karachi 75270, Pakistan
}

Correspondence should be addressed to Najeeb Alam Khan, njbalam@yahoo.com

Received 21 October 2011; Accepted 13 November 2011

Academic Editors: P. Roy and W.-H. Steeb

Copyright (c) 2012 Najeeb Alam Khan et al. This is an open access article distributed under the Creative Commons Attribution License, which permits unrestricted use, distribution, and reproduction in any medium, provided the original work is properly cited.

We construct the approximate solutions of the time-fractional Schrödinger equations, with zero and nonzero trapping potential, by homotopy analysis method (HAM). The fractional derivatives, in the Caputo sense, are used. The method is capable of reducing the size of calculations and handles nonlinear-coupled equations in a direct manner. The results show that HAM is more promising, convenient, efficient and less computational than differential transform method (DTM), and easy to apply in spaces of higher dimensions as well.

\section{Introduction}

The theory of derivatives of fractional (nonintegers) orders stimulates considerable interest in the areas of mathematics, physics, engineering, and other sciences. Fractional derivatives [1-5] provide an excellent tool for the description of memory and hereditary properties of various material and processes. The beauty of this subject is that a fractional derivative is not a local point property. This considers the history and nonlocal distributed effects. Perhaps, this subject translates the reality of nature better. Application of fractional calculus are found in different areas of sciences such as physics, continuum mechanics, signal processing, electromagnetics, and bioengineering. The electrical properties of nerve cell membranes and the propagation of electrical signals are well characterized by differential equations of fractional order. The fractional differential equations (FDE) [6-11] appear more and more frequently in different research areas and engineering applications. Exact solution of 
nonlinear partial differential equations has become one of the central themes of perpetual interest in mathematical physics. In order to better understand these phenomena as well as further apply them in the practical life, it is important to seek their exact solutions.

A great deal of effort has also been expanded in attempting to find robust and stable numerical and analytical methods for solving fractional differential equations of physical interest. These methods include Laplace transform method, Fourier transform method, finite difference method [12], fractional linear multistep methods, Adomian decomposition method (ADM) [13], variational iteration method (VIM) [14], homotopy perturbation method (HPM) [15], differential transform method [16], and homotopy analysis method (HAM) [17-20].

The aim of this paper is to solve the Schrödinger equation with fractional order using the homotopy analysis method. By introducing an embedding parameter $q$ the nonlinear fractional differential equation is converted to a linear fractional differential equation at $q=0$. When $q$ evolves, the differential equation becomes the original one at $q=1$. The method has been used in a variety of problems and the details can be found in Liao's book [17]. The method gives rapidly convergent successive approximations of the exact solution if such solution exists; otherwise, a few approximations can be used for numerical purposes. Wang [12] presented the numerical solution Schrödinger equations by means of finite difference scheme. Khuri [13] applied ADM to obtain the solution of cubic Schrödinger equations. Wazwaz [14] presented the exact solution of the linear and nonlinear onedimensional Schrödinger equations by VIM. Recently, Ravi Kanth [16] and his colleague presented the exact solution of the linear and nonlinear Schrödinger equations by differential transformation method (DTM).

The aim of this paper is to investigate the approximate solutions of the time-fractional Schrödinger equations, with zero and non-zero trapping potential, by means of HAM. The convergent region is then obtained by looking at the real and imaginary parts of the series in plot.

\section{Fractional Schrödinger Equations and Preliminaries}

The time-fractional Schrödinger equations (FSE) has the following form:

$$
\begin{gathered}
i \frac{\partial^{\alpha} \psi(X, t)}{\partial t^{\alpha}}+\frac{1}{2} \nabla^{2} \psi(X, t)-V_{d}(X) \psi-\beta_{d}|\psi|^{2} \psi=0 ; \quad t \geq 0 \\
\psi(X, 0)=\psi_{0}(X), \quad X \in \mathfrak{R}^{d}
\end{gathered}
$$

where $V_{d}(X)$ is the trapping potential and $\beta_{d}$ is a real constant. The physical model (2.1) and its generalized forms occur in various areas of physics, including nonlinear optics, plasma physics, superconductivity, and quantum mechanics.

We give some basic definitions, notations, and properties of the fractional calculus theory, which will be used later in this work. 
Definition 2.1. The Riemann-Liouville fractional integral operator of order $\mu$ on the usual Lebesgue space $L_{1}[a, b]$ is given by

$$
\begin{gathered}
J^{\mu} f(x)=\frac{1}{\Gamma(\mu)} \int_{0}^{x}(x-t)^{\mu-1} f(t) d t ; \quad \mu>0 \\
J^{0} f(x)=f(x)
\end{gathered}
$$

This integral operator has the following properties:

(i) $J^{\alpha} J^{\beta}=J^{\alpha+\beta}=J^{\beta} J^{\alpha}, \quad \alpha, \beta>0$,

(ii) $J^{\alpha}(x-a)^{\gamma}=\frac{\Gamma(\gamma+1)}{\Gamma(\alpha+\gamma+1)}(x-a)^{\alpha+\gamma}, \quad \alpha>0, \gamma>-1$.

Definition 2.2. The Caputo definition of fractal derivative operator is given by

$$
D^{\mu} f(x)=\frac{1}{\Gamma(m-\mu)} \int_{0}^{t}(x-\tau)^{m-\mu-1} f^{(m)}(\tau) d \tau \quad m-1<\mu \leq m, \quad m \in N, \quad x>0 .
$$

It has the following two basic properties:

$$
D^{\mu} J^{\mu} f(x)=f(x)
$$

and

$$
J^{\mu} D^{\mu} f(x)=f(x)-\sum_{k=0}^{m-1} f^{(k)}\left(0^{+}\right) \frac{(x)^{k}}{k !}, \quad x>0
$$

The Caputo fractional derivative is considered here because it allows traditional initial and boundary conditions to be included in the formulation of the problem.

\section{Basic Idea of HAM}

In this paper, we apply the HAM to the linear and nonlinear problems to be discussed. In order to show the basic idea of HAM, consider the following nonlinear fractional differential equation:

$$
\mathcal{N}_{\mp}[\psi(X, t ; q)]=0
$$

where $\mathcal{N}_{\mp}$ is a nonlinear fractional operator, $X$ and $t$ denote the independent variables, and $\psi$ is an unknown function. By means of the HAM, we first construct the so-called zeroth-order deformation equation

$$
(1-q) £_{\mp}\left[\phi(X, t ; q)-\psi_{0}(X, t)\right]=q \hbar \mathcal{N}_{\mp}[\phi(X, t ; q)]
$$


where $q \in[0,1]$ is the embedding parameter, $\hbar \neq 0$ is an auxiliary parameter, $£_{\bar{q}}$ is an auxiliary linear operator, $\phi(X, t ; q)$ are unknown functions, and $\psi_{0}(X, t)$ are initial guesses of $\phi(X, t ; q)$. It is obvious that for $q=0$ and $q=1$, (3.3) becomes

$$
\phi(X, t ; 0)=\psi_{0}(X, t), \quad \phi(X, t ; 1)=\psi(X, t),
$$

respectively. Thus as $q=0$ increases from 0 to 1 , the solution $\phi(X, t ; q)$ varies from the initial guess $\psi_{0}(X, t)$ to the exact solution $\psi(X, t)$. Expanding $\phi(X, t ; q)$ in Taylor series with respect to $q$, one has

$$
\phi(X, t ; q)=\psi_{0}(X, t)+\sum_{m=1}^{+\infty} \psi_{m}(X, t) q^{m}
$$

where

$$
\psi_{m}(X, t)=\left.\frac{1}{m !} \frac{\partial^{m} \phi(X, t ; q)}{\partial q^{m}}\right|_{q=0}
$$

The convergence of the series (3.4) depends upon the auxiliary parameter $\hbar$. If it is convergent at $q=1$, one has

$$
\psi(X, t)=\psi_{0}(X, t)+\sum_{m=1}^{+\infty} \psi_{m}(X, t)
$$

which must be one of the solutions of the original nonlinear equations, as proved by Liao [17]. Define the vectors

$$
\overrightarrow{\psi_{n}}=\left\{\psi_{0}(X, t), \psi_{1}(X, t), \psi_{2}(X, t), \ldots, \psi_{n}(X, t)\right\}
$$

Differentiating the zeroth-order deformation (3.2) $m$-times with respect to $q$ and then dividing them by $m !$, and finally setting $q=0$, we get the following $m$ th-order deformation equation:

$$
£_{\mp}\left[\psi_{m}(X, t)-\chi_{m} \psi_{m-1}(X, t)\right]=\hbar R_{m}\left(\vec{\psi}_{m-1}\right)
$$

where

$$
R_{m}\left(\vec{\psi}_{m-1}\right)=\left.\frac{1}{(m-1) !} \frac{\partial^{m-1} \mathcal{N}_{\mathcal{F}}[\phi(X, t ; q)]}{\partial q^{m-1}}\right|_{q=0,} \quad X_{m}= \begin{cases}0 ; & m=1 \\ 1 ; & m>1\end{cases}
$$

It should be emphasized that $\psi_{m}(X, t ; q)$ for $m \geq 1$ is governed by the linear equations (3.8) with boundary conditions that come from the original problem, which can be solved by the symbolic computation software MATHEMATICA. The success of the technique is based on the proper selection of the initial guess. 


\section{Implementation of the Method}

In this section, we introduce the above reliable approach, in a realistic and efficient way, to handle nonlinear Schrödinger equation with time-fractional derivative.

\subsection{Nonlinear-Time Fractional Schrödinger Equation (NLFSE)}

Setting $\psi(X, t)=v(X, t)+i w(X, t)$ and $\psi(X, 0)=v(X, 0)+i w(X, 0)$ in (2.1) leads to the following coupled system of equations

$$
\begin{gathered}
D^{\alpha} v+\frac{1}{2} \nabla^{2} w-V_{d} w-\beta_{d}\left(w v^{2}+w^{3}\right)=0, \\
D^{\alpha} w-\frac{1}{2} \nabla^{2} v-V_{d} v+\beta_{d}\left(v^{3}+w^{2} v\right)=0,
\end{gathered}
$$

subject to the initial conditions

$$
v(X, 0)=v_{0}=F(X), \quad w(X, 0)=w_{0}=G(X) .
$$

Equations (4.1) and (4.3) suggest that we define the nonlinear operator as

$$
\begin{aligned}
\mathcal{N}_{\mp}^{1}\left[\varphi_{1}(X, t ; q), \varphi_{2}(X, t ; q)\right]= & D^{\alpha} \varphi_{1}(X, t ; q)+\frac{1}{2} \nabla^{2} \varphi_{2}(X, t ; q)-V_{d} \varphi_{2}(X, t ; q) \\
& -\varphi_{2}(X, t ; q) \varphi_{1}^{2}(X, t ; q)-\varphi_{2}^{3}(X, t ; q), \\
\mathcal{N}_{\mp}^{2}\left[\varphi_{1}(X, t ; q), \varphi_{2}(X, t ; q)\right]= & D^{\alpha} \varphi_{2}(X, t ; q)-\frac{1}{2} \nabla^{2} \varphi_{1}(X, t ; q)+V_{d} \varphi_{1}(X, t ; q) \\
& +\varphi_{1}^{3}(X, t ; q)+\varphi_{2}^{2}(X, t ; q) \varphi_{1}(X, t ; q),
\end{aligned}
$$

and the linear operator

$$
\begin{gathered}
£_{\bar{q}}[\phi(X, t ; q)]=D^{\alpha}[\phi(X, t ; q)] \text { with the property } £_{\bar{q}}\left[c_{1}(X)\right]=0 \\
£_{\mathscr{q}}\left[v_{m}(X, t)-\chi_{m} v_{m-1}(X, t)\right]=\hbar R_{1, m}\left[\vec{v}_{m-1}, \vec{w}_{m-1}\right] \\
£_{\bar{q}}\left[w_{m}(X, t)-X_{m} w_{m-1}(X, t)\right]=\hbar R_{2, m}\left[\vec{v}_{m-1}, \vec{w}_{m-1}\right]
\end{gathered}
$$

where

$$
\begin{aligned}
R_{1, m}\left[\vec{v}_{m-1}, \vec{w}_{m-1}\right]= & D^{\alpha} v_{m-1}+\frac{1}{2} \nabla^{2} w_{m-1}-V_{d} w_{m-1} \\
& -\beta_{d}\left(\sum_{k=0}^{m-1} v_{m-k-1} \sum_{l=0}^{k} v_{k-l} w_{l}+\sum_{k=0}^{m-1} w_{m-k-1} \sum_{l=0}^{k} w_{k-l} w_{l}\right) \\
R_{2, m}\left[\vec{v}_{m-1}, \vec{w}_{m-1}\right]= & D^{\alpha} w_{m-1}-\frac{1}{2} \nabla^{2} v_{m-1}+V_{d} v_{m-1} \\
& +\beta_{d}\left(\sum_{k=0}^{m-1} w_{m-k-1} \sum_{l=0}^{k} w_{k-l} v_{l}+\sum_{k=0}^{m-1} v_{m-k-1} \sum_{l=0}^{k} v_{k-l} \boldsymbol{v}_{l}\right) .
\end{aligned}
$$


Obviously, the solution of the $m$ th-order deformation (4.8) for $m \geq 1$ becomes

$$
\begin{aligned}
v_{m}(X, t) & =\chi_{m} v_{m-1}(X, t)+\hbar J^{\alpha} R_{1, m}\left[\vec{v}_{m-1}, \vec{w}_{m-1}\right] \\
w_{m}(X, t) & =\chi_{m} w_{m-1}(X, t)+\hbar J^{\alpha} R_{2, m}\left[\vec{v}_{m-1}, \vec{w}_{m-1}\right]
\end{aligned}
$$

Example 4.1. We first consider the one-dimensional NLFSE with zero trapping potential (i.e., $V_{d}(x)=0$ ) and $\beta_{d}=-1$

$$
i D_{t}^{\alpha} \psi(x, t)+\frac{1}{2} \frac{\partial^{2} \psi(x, t)}{\partial x^{2}}+|\psi|^{2} \psi=0, \quad t \geq 0,0<\alpha \leq 1
$$

subject to the initial condition $\psi(x, 0)=e^{i x}$.

Solving the above equations

$$
\begin{gathered}
v_{0}=\cos x, \quad w_{0}=\sin x \\
v_{1}=\frac{\hbar t^{\alpha} \sin x}{2 \Gamma(\alpha+1)}, \quad w_{2}=\frac{-\hbar t^{\alpha} \cos x}{2 \Gamma(\alpha+1)} \\
v_{2}=\frac{\hbar(\hbar+1) t^{\alpha} \sin x}{2 \Gamma(\alpha+1)}-\frac{\hbar^{2} t^{2 \alpha} \cos x}{4 \Gamma(2 \alpha+1)}, \quad w_{2}=\frac{-\hbar(\hbar+1) t^{\alpha} \cos x}{2 \Gamma(\alpha+1)}-\frac{\hbar^{2} t^{2 \alpha} \sin x}{4 \Gamma(2 \alpha+1)} \\
v_{3}=\left(\frac{\hbar(\hbar+1)^{2} t^{\alpha}}{2 \Gamma(\alpha+1)}-\frac{\hbar^{3} t^{3 \alpha}\left(5(\Gamma(\alpha+1))^{2}-2 \Gamma(2 \alpha+1)\right)}{8(\Gamma(\alpha+1))^{2} \Gamma(3 \alpha+1)}\right) \sin x-\frac{\hbar^{2}(\hbar+1) t^{2 \alpha} \cos x}{2 \Gamma(2 \alpha+1)} \\
w_{3}=\left(\frac{-\hbar(\hbar+1)^{2} t^{\alpha}}{2 \Gamma(\alpha+1)}+\frac{\hbar^{3} t^{3 \alpha}\left(5(\Gamma(\alpha+1))^{2}-2 \Gamma(2 \alpha+1)\right)}{8(\Gamma(\alpha+1))^{2} \Gamma(3 \alpha+1)}\right) \cos x-\frac{\hbar^{2}(\hbar+1) t^{2 \alpha} \sin x}{2 \Gamma(2 \alpha+1)}
\end{gathered}
$$

and so on, in this manner the rest of the components can be obtained. Therefore, the approximate solution is

$$
\psi(x, t)=\sum_{n=0}^{7}\left(v_{n}+i w_{n}\right)
$$

The exact solution of (4.10) for $\alpha=1$ is $\psi=e^{i(x+(t / 2))}$. When $\hbar=-1, \alpha=1$, the solution obtained by [13-16] is recovered as a special case.

Example 4.2. Consider the one-dimensional NLFSE with trapping potential, that is, $V_{d}(x)=$ $\cos ^{2} x$ and $\beta_{d}=-1$

$$
i D_{t}^{\alpha} \psi(x, t)+\frac{1}{2} \frac{\partial^{2} \psi(x, t)}{\partial x^{2}}-\psi \cos ^{2} x-|\psi|^{2} \psi=0, \quad t \geq 0,0<\alpha \leq 1 .
$$

subject to the initial condition $\psi(x, 0)=\sin x$. 
Solving above equations we obtain the solution in a series form

$$
\begin{gathered}
v_{0}=\sin x, \quad w_{0}=0 \\
v_{1}=0, \quad w_{1}=\frac{3 \hbar t^{\alpha} \sin x}{2 \Gamma(\alpha+1)} \\
v_{2}=-\frac{9 \hbar^{2} t^{2 \alpha} \sin x}{4 \Gamma(2 \alpha+1)}, \quad w_{2}=\frac{3 \hbar(\hbar+1) t^{\alpha} \sin x}{2 \Gamma(\alpha+1)} \\
v_{3}=-\frac{9 \hbar^{2}(\hbar+1) t^{2 \alpha} \sin x}{2 \Gamma(2 \alpha+1)}, \\
\frac{3 \hbar(\hbar+1)^{2} t^{\alpha} \sin x}{2 \Gamma(\alpha+1)}+\frac{9 \hbar^{3} t^{3 \alpha} \sin x\left((-5+2 \cos x)(\Gamma(\alpha+1))^{2}+2 \Gamma(2 \alpha+1) \sin ^{2} x\right)}{8(\Gamma(\alpha+1))^{2} \Gamma(3 \alpha+1)}, \\
\psi(x, t)=\sum_{n=0}^{7}\left(v_{n}+i w_{n}\right) .
\end{gathered}
$$

The exact solution of (4.13) for $\alpha=1$ is $u=\sin x e^{-3 i t / 2}$. When $\hbar=-1, \alpha=1$, the solution obtained by [13-16] is recovered as a special case.

Example 4.3. Consider the two dimensional NLFSE with trapping potential

$$
\begin{gathered}
i D^{\alpha} \psi+\frac{1}{2}\left(\frac{\partial^{2} \psi}{\partial x^{2}}+\frac{\partial^{2} \psi}{\partial y^{2}}\right)+V_{d}(x, y) \psi+\beta_{d}|\psi|^{2} \psi=0, \\
t \geq 0, \quad 0<\alpha \leq 1, \quad(x, y) \in[0,2 \pi] \times[0,2 \pi] .
\end{gathered}
$$

Here $V_{d}(x, y)=1-\sin ^{2} x \sin ^{2} y, \beta_{d}=1$, subject to the initial condition $\psi(x, y, 0)=\sin x \sin y$. Solving the above equation we obtain the approximate solution in a series form

$$
\begin{gathered}
v_{0}=\sin x \sin y, \quad w_{0}=0 \\
v_{1}=0, \quad w_{1}=\frac{2 \hbar t^{\alpha} \sin x \sin y}{\Gamma(\alpha+1)} \\
v_{2}=\frac{4 \hbar^{2} t^{2 \alpha} \sin x \sin y}{\Gamma(2 \alpha+1)}, \quad w_{2}=\frac{2 \hbar(\hbar+1) t^{\alpha} \sin x \sin y}{\Gamma(\alpha+1)} \\
v_{3}=\frac{-8 \hbar^{2}(\hbar+1) t^{2 \alpha} \sin x \sin y}{\Gamma(2 \alpha+1)}
\end{gathered}
$$




$$
\begin{gathered}
w_{3}=-\frac{4 \hbar^{3} t^{3 \alpha} \sin x \sin y\left(2\left(1+\sin ^{2} x \sin ^{2} y\right)(\Gamma(\alpha+1))^{2}-\Gamma(2 \alpha+1) \sin ^{2} x \sin ^{2} y\right)}{(\Gamma(\alpha+1))^{2} \Gamma(3 \alpha+1)} \\
+\frac{2 \hbar(\hbar+1)^{2} t^{\alpha} \sin x \sin y}{\Gamma(\alpha+1)}, \\
\psi(x, y, t)=\sum_{n=0}^{7}\left(v_{n}+i w_{n}\right) .
\end{gathered}
$$

The exact solution of (4.15) for $\hbar=-1, \alpha=1$ is $\psi=\sin x \sin y e^{-2 i t}$.

Example 4.4. Consider the three-dimensional NLFSE with trapping potential

$$
\begin{gathered}
i D^{\alpha} \psi+\frac{1}{2}\left(\frac{\partial^{2} \psi}{\partial x^{2}}+\frac{\partial^{2} \psi}{\partial y^{2}}+\frac{\partial^{2} \psi}{\partial z^{2}}\right)-V_{d}(x, y, z) \psi-|\psi|^{2} \psi=0, \quad t \geq 0, \quad 0<\alpha \leq 1, \\
(x, y, z) \in[0,2 \pi] \times[0,2 \pi] \times[0,2 \pi]
\end{gathered}
$$

where $V_{d}(x, y, z)=1-\sin ^{2} x \sin ^{2} y \sin ^{2} z$ subject to the initial condition

$$
\psi(x, y, z, 0)=\sin x \sin y \sin z .
$$

Solving the above equations,

$$
\begin{gathered}
v_{0}=\sin x \sin y \sin z, w_{0}=0 \\
v_{1}=0, \quad w_{1}=\frac{5 \hbar t^{\alpha} \sin x \sin y \sin z}{2 \Gamma(\alpha+1)} \\
v_{2}=\frac{-25 \hbar^{2} t^{2 \alpha} \sin x \sin y \sin z}{4 \Gamma(2 \alpha+1)}, \quad w_{2}=\frac{-5 \hbar(\hbar+1) t^{\alpha} \sin x \sin y \sin z}{2 \Gamma(\alpha+1)} .
\end{gathered}
$$

Finally the approximate solution in the series form is

$$
\psi(x, y, z, t)=\sum_{n=0}^{7}\left(v_{n}+i w_{n}\right)
$$

The exact solution of (4.17) for $\alpha=1$ is $\psi=\sin x \sin y \sin z e^{-5 i t / 2}$.

\section{Closing Comments}

The basic goal of this work has been to extend the works made in the nonlinear physical problem to construct solutions for nonlinear Schrödinger equation with time-fractional derivatives. The goal has been achieved and new solutions of have been derived for nonlinear equations with time fractional derivatives. The proposed approach works successfully 


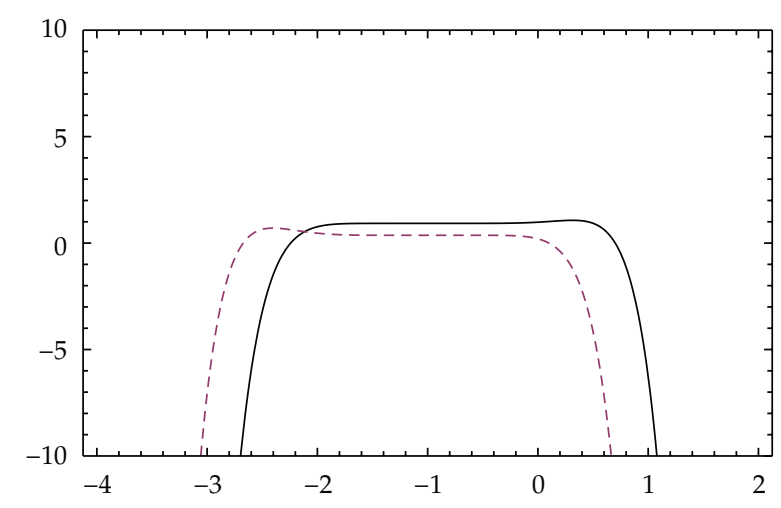

Figure 1: The $\hbar$-curve for Example $4.1 x=0.2, t=0.2, \alpha=0.7$.

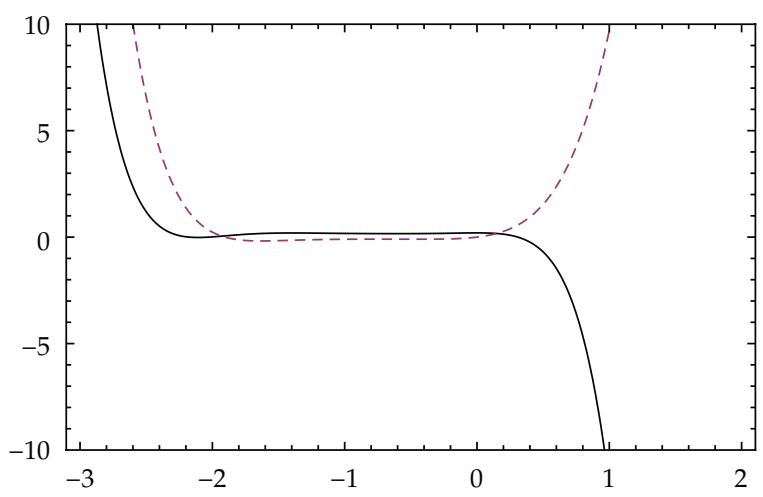

Figure 2: The $\hbar$-curve for Example $4.2 x=0.2, t=0.2, \alpha=0.7$.

in handling nonlinear fractional Schrödinger equations directly with a minimum size of calculations.

Borhanifer and Abazari [11] applied the differential transformation method (DTM) to solving Schrödinger and coupled Schrödinger equations. The major lacks of DTM are that it requires transformation, and the given differential equation and related initial conditions are transformed into a recurrence equation that finally leads to the solution of a system of algebraic equations as coefficients of a power series solution. The main disadvantage of DTM is that it requires transformation, which will be complicated and computational cost will be too much. This emphasizes the fact that the presented approach can be used in a wider class of system of nonlinear fractional differential equations. HAM is a powerful and efficient technique in finding exact and approximate solutions for linear and nonlinear models. HAM provides more realistic solutions that converge very rapidly in real physical problems. The numerical examples show that the solutions are in good agreement with their respective exact solutions for $\alpha=1$.

In the last, we present the $\hbar$-curves to see the convergent region of $\hbar$ as in Figures 1-4. We plot the imaginary and real part of each example in the same figure, and the convergent region is the region of intersection between the convergent regions of the imaginary and real parts. In all figures $\hbar=-1$ is in the convergent region. 


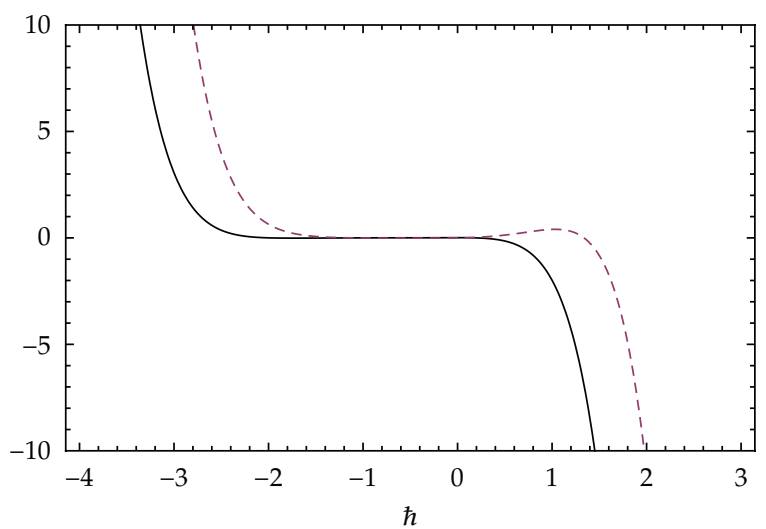

Figure 3: The $\hbar$-curve for Example $4.3 x=0.1, y=0.1, t=0.5, \alpha=0.9$.

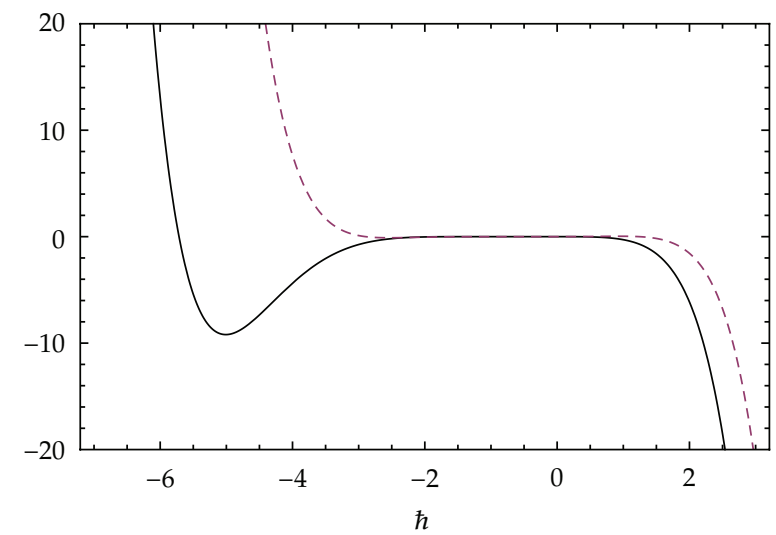

Figure 4: The $\hbar$-curve for Example $4.4 x=0.1, y=0.1, z=0.1, t=0.5, \alpha=0.9$.

\section{Acknowledgments}

The author N. A. Khan is thankful and grateful to the dean of the Faculty of Sciences, University of Karachi, Pakistan for supporting and facilitating this research work. The author M. Jamil is highly thankful and grateful to the Abdul Salam School of Mathematical Sciences, GC University, Lahore, Pakistan, Department of Mathematics, NED University of Engineering and Technology, Pakistan, and also Higher Education Commission of Pakistan for generous support and facilitating this research work.

\section{References}

[1] A. Carpinteri and F. Mainardi, Fractals Fractional Calculus in Continuum Mechanics, Springer, New York, NY, USA, 1997.

[2] K. S. Miller and B. Ross, An Introduction to the Fractional Calculus and Fractional Differential Equations, Wiley, New York, NY, USA, 1993.

[3] K. B. Oldham and J. Spanier, The Fractional Calculus, Academic Press, New York, NY, USA, 1974.

[4] I. Podlubny, Fractional Differential Equations, Acaddemic Press, San Diego, Calif, USA, 1999. 
[5] K. Diethelm, "An algorithm for the numerical solution of differential equations of fractional order," Electronic Transactions on Numerical Analysis, vol. 5, pp. 1-6, 1997.

[6] J. Cang, Y. Tan, H. Xu, and S. J. Liao, "Series solutions of non-linear Riccati differential equations with fractional order," Chaos, Solitons and Fractals, vol. 40, no. 1, pp. 1-9, 2009.

[7] N. A. Khan, A. Ara, S. A. Ali, and A. Mahmood, "Analytical study of Navier-Stokes equation with fractional orders using He's homotopy perturbation and variational iteration methods," International Journal of Nonlinear Sciences and Numerical Simulation, vol. 10, no. 9, pp. 1127-1134, 2009.

[8] N. A. Khan, A. Ara, and A. Mahmood, "Approximate solution of time-fractional chemical engineering equations: a comparative study," International Journal of Chemical Reactor Engineering, vol. 8, article A19, 2010.

[9] A. Mahmood, S. Parveen, A. Ara, and N. A. Khan, "Exact analytic solutions for the unsteady flow of a non-Newtonian fluid between two cylinders with fractional derivative model," Communications in Nonlinear Science and Numerical Simulation, vol. 14, no. 8, pp. 3309-3319, 2009.

[10] A. Mahmood, C. Fetecau, N. A. Khan, and M. Jamil, "Some exact solutions of the oscillatory motion of a generalized second grade fluid in an annular region of two cylinders," Acta Mechanica Sinica, vol. 26, no. 4, pp. 541-550, 2010.

[11] A. Borhanifar and R. Abazari, "Numerical study of nonlinear Schrödinger and coupled Schrödinger equations by differential transformation method," Optics Communications, vol. 283, no. 10, pp. 20262031, 2010.

[12] H. Wang, "Numerical studies on the split-step finite difference method for nonlinear Schrödinger equations," Applied Mathematics and Computation, vol. 170, no. 1, pp. 17-35, 2005.

[13] S. Khuri, "A new approach to the cubic Schrödinger equation: an application of the decomposition technique," Applied Mathematics and Computation, vol. 97, pp. 251-254, 1998.

[14] A. M. Wazwaz, "A study on linear and nonlinear Schrodinger equations by the variational iteration method," Chaos, Solitons and Fractals, vol. 37, no. 4, pp. 1136-1142, 2008.

[15] A. Sadighi and D. D. Ganji, "Analytic treatment of linear and nonlinear Schrödinger equations: a study with homotopy-perturbation and Adomian decomposition methods," Physics Letters A, vol. 372, no. 4, pp. 465-469, 2008.

[16] A. S. V. Ravi Kanth and K. Aruna, "Two-dimensional differential transform method for solving linear and non-linear Schrödinger equations," Chaos, Solitons and Fractals, vol. 41, no. 5, pp. 2277-2281, 2009.

[17] S. J. Liao, Beyond Perturbation: Introduction to the Homotopy Analysis Method, Chapman and Hall/CRC Press, Boca Raton, 2003.

[18] S. J. Liao and K. F. Cheung, "Homotopy analysis of nonlinear progressive waves in deep water," Journal of Engineering Mathematics, vol. 45, no. 2, pp. 105-116, 2003.

[19] L. Song and H. Zhang, "Application of homotopy analysis method to fractional KdV-BurgersKuramoto equation," Physics Letters A, vol. 367, no. 1-2, pp. 88-94, 2007.

[20] N. A. Khan, A. Ara, M. Afzal, and A. Khan, "Analytical aspect of fourth-order parabolic partial differential equations with variable coefficients," Mathematical and Computational Applications, vol. 15, no. 3, pp. 481-489, 2010. 


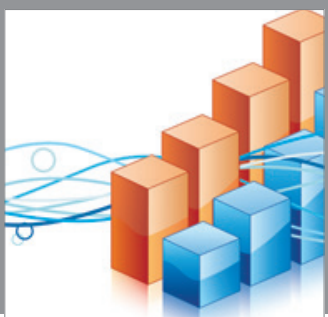

Advances in

Operations Research

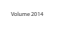

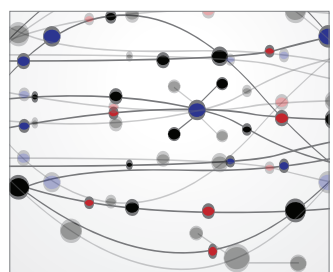

\section{The Scientific} World Journal
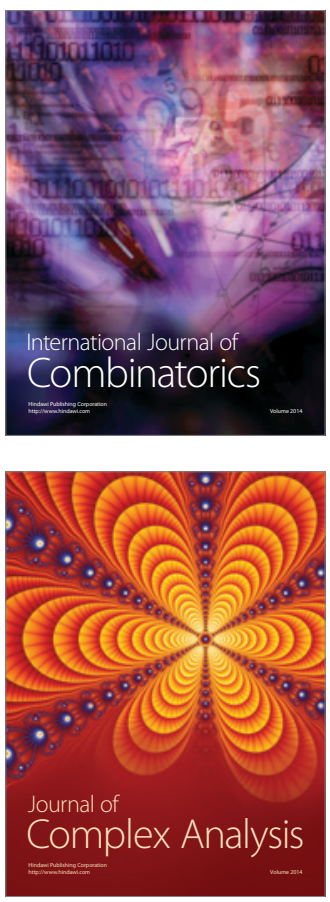

International Journal of

Mathematics and

Mathematical

Sciences
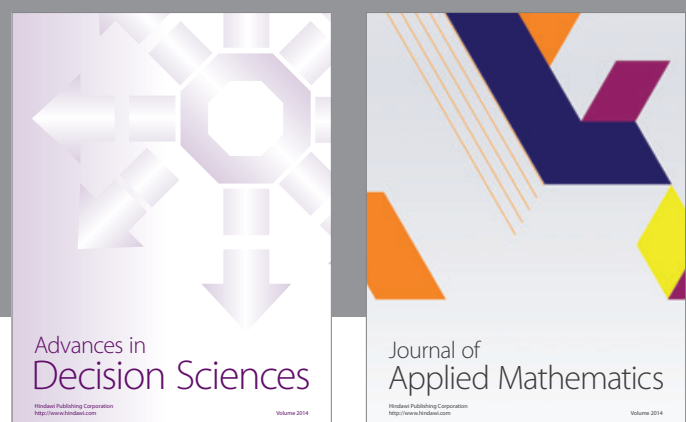

Journal of

Applied Mathematics
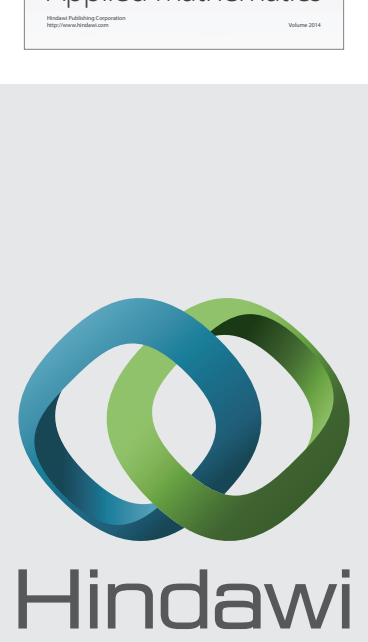

Submit your manuscripts at http://www.hindawi.com
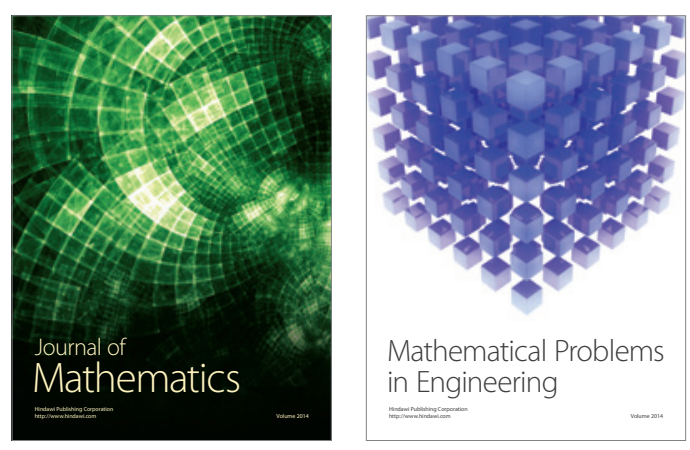

Mathematical Problems in Engineering
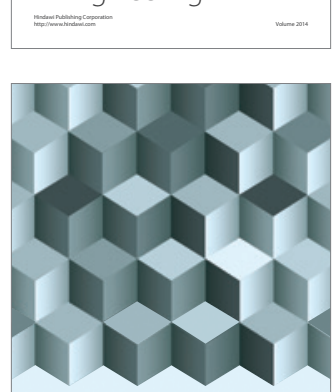

Journal of

Function Spaces
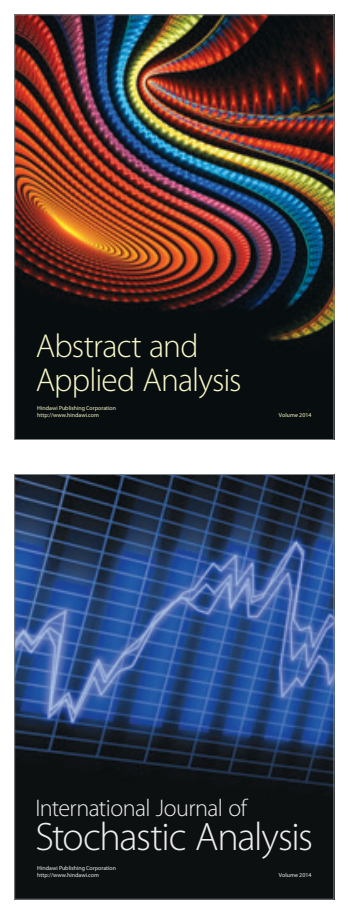

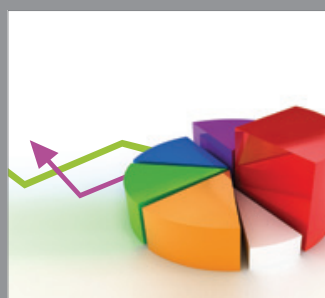

ournal of

Probability and Statistics

Promensencen
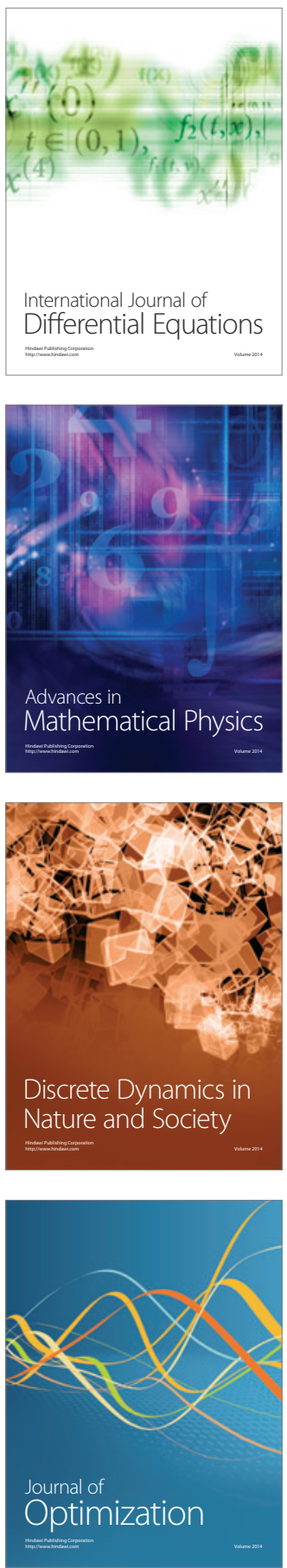